\title{
Evolution of shapes in even-even nuclei using the standard interacting boson model
}

\author{
Ali M. Khalaf • Mahmoud M. Taha
}

Received: 4 July 2014 / Accepted: 4 February 2015 / Published online: 18 February 2015

(C) The Author(s) 2015. This article is published with open access at Springerlink.com

\begin{abstract}
The sd-version of the interacting boson model (IBM) is used to establish the shape phase transitional structure. A simplified Hamiltonian is used which is intermediate between the three dynamical symmetries of $\mathrm{U}(6)$, namely the spherical $\mathrm{U}(5)$, the prolate and oblate deformed SU(3) and the $\gamma$-unstable $\mathrm{O}(6)$ limits. The potential energy surfaces (PESs) to the IBM Hamiltonian have been obtained using the intrinsic state formalism which introduces the shape variables $\beta$ and $\gamma$. The Gadolinium $(\mathrm{Gd})$ and Ruthenium $(\mathrm{Ru})$ isotopic chains have been taken as examples in illustrating the U(5)-SU(3) and U(5)$\mathrm{O}(6)$ shape phase transitions, respectively. We used the standard $\chi^{2}$ test to get the IBM Hamiltonian parameters. The fit is performed by minimizing the $\chi^{2}$ function for some selected experimental low-lying energy levels, the two neutron separation energies and $\mathrm{B}(\mathrm{E} 2)$ transition rates.
\end{abstract}

Keywords Nuclear structure - Potential energy surface · Interacting boson model

\section{Introduction}

The interacting boson model (IBM) [1] was designed to describe the collective quadrupole degrees of freedom

A. M. Khalaf

Physics Department, Faculty of Science, Al-Azhar University, Cairo, Egypt

M. M. Taha (ه)

Mathematics and Theoretical Physics Department, Nuclear Research Center, Atomic Energy Authority, P.No. 13759, Cairo, Egypt

e-mail: mahmoudmt@hotmail.com in nuclei. The IBM Hamiltonian was written from the beginning in second quantization form in terms of the generators of the unitary Lie algebra U(6) subtended by $\mathrm{s}$ and $\mathrm{d}$ bosons which carry angular momentum 0 and 2, respectively. The model present three special limits that can be solved easily. These three limits are U(5), SU(3) and $\mathrm{O}(6)$ dynamical symmetries appropriate for an harmonic vibrator, axial deformed rotor and $\gamma$-unstable deformed rotor, respectively. Each of these limits is assigned to spherical, axially deformed and deformed with $\gamma$-instability shapes, respectively. Among the transitional Hamiltonian a specially interesting case occurs when it describes a critical point in the transition from a given shape to another. A shape phase transition may be of first or second order, depending on the discontinuity of the first- or second-order derivatives of the order parameter as a function of the control parameters [2-10].

The classical limit of the IBM is defined as its expectation value in a coherent state [10-12] and yields a function of shape variables $\beta$ and $\gamma$ which can be interpreted as a potential energy surface (PES) depending on these parameters. The symmetry $\mathrm{E}(5)$ [13] is designed for the critical point of the transition from spherical to deformed $\gamma$-unstable shapes. Later $[14,15] \mathrm{X}(5)$ and $\mathrm{Y}(5)$ describe the critical points between spherical and axially deformed shapes and between axial and triaxial deformed shapes, respectively.

In the present work, we use the two parameters sd-IBM1 Hamiltonian with the intrinsic coherent state method to produce the potential energy surfaces (PESs) for chains of isotopes ${ }^{150-162} \mathrm{Gd}$ and ${ }^{96-114} \mathrm{Ru}$ involving nuclei suggested [16-18] to be good candidates for $\mathrm{X}(5)$ and $\mathrm{E}(5)$ critical point symmetries. 
A simplified Hamiltonian of the sd-IBM in the intrinsic condensate state

In this work, we adopt a simplified two-parameter IBM-1 Hamiltonian in the form

$H=\epsilon \hat{n}_{d}+a_{2} \hat{Q}(\chi) \cdot \hat{Q}(\chi)$

where the $d$-boson number operator $\hat{n}_{d}$ and the quadrupole operator $Q(\chi)$ are defined as

$\hat{n}_{d}=d^{\dagger} \cdot \tilde{d}$

$\hat{Q}(\chi)=s^{\dagger} \tilde{d}+d^{\dagger} \tilde{s}+\chi\left[d^{\dagger} \times \tilde{d}\right]^{(2)}$

with $\chi$ the structure parameter of the quadrupole operator of the IBM, and $\left[d^{\dagger} \times \tilde{d}\right]^{(2)}$ stands for the $l=2$ tensor coupling of the d-boson creation and annihilation operators $\left(\tilde{d}_{\mu}=\right.$ $\left.(-1)^{\mu} d_{-\mu}\right)$, where $\mu=-2,-1,0,+1,+2$ is the angular momentum projection, and $(\cdot)$ denoting the scalar product.

The geometric interpretation of the Hamiltonian (1) can be derived using the intrinsic condensate state $|N \beta \gamma\rangle$ defined by $[10,11]$

$$
|N \beta \gamma\rangle=\frac{1}{\sqrt{N !}}\left(\Gamma_{c}^{\dagger}\right)^{N}|0\rangle
$$

with

$$
\Gamma_{c}^{\dagger}=\frac{1}{\sqrt{1+\beta^{2}}}\left[s^{\dagger}+\beta \cos \gamma d_{0}^{\dagger}+\frac{1}{\sqrt{2}} \sin \gamma\left(d_{2}^{\dagger}+d_{-2}^{\dagger}\right)\right]
$$

where $\beta$ and $\gamma$ are the geometric parameters. We get the ground state potential energy surface (PES) by calculating the expectation value of the Hamiltonian on the intrinsic boson condensate state:

$$
\begin{aligned}
E(N, \beta, \gamma, \chi)= & \langle N \beta \gamma|H| N \beta \gamma\rangle \\
= & \epsilon \frac{N \beta^{2}}{1+\beta^{2}}+a_{2}\left\{\frac{N}{1+\beta^{2}}\left[5+\left(1+\chi^{2}\right) \beta^{2}\right]\right. \\
& \left.+\frac{N(N-1)}{\left(1+\beta^{2}\right)^{2}}\left[4 \beta^{2}-4 \sqrt{\frac{2}{7}} \chi \beta^{3} \cos 3 \gamma+\frac{2}{7} \chi^{2} \beta^{4}\right]\right\}
\end{aligned}
$$

If we eliminate the contributions of the one-body terms of the quadrupole-quadrupole interaction, then

$$
E(N, \beta, \gamma, \chi)=\frac{A_{2} \beta^{2}+A_{3} \beta^{3} \cos 3 \gamma+A_{4} \beta^{4}}{\left(1+\beta^{2}\right)^{2}}
$$

with

$$
\begin{aligned}
& A_{2}=\left[\epsilon+4 a_{2}(N-1)\right] N \\
& A_{3}=-4 \sqrt{\frac{2}{7}} \chi a_{2}(N-1) N \\
& A_{4}=\left[\epsilon+\frac{2}{7} \chi^{2}(N-1) a_{2}\right] N
\end{aligned}
$$

Minimization of the PES with respect to $\beta$ for a given value of $\chi$, gives the equilibrium value $\beta_{m}$ defining phase of the system; $\beta_{m}=0$ corresponding to the symmetric phase while $\beta_{m} \neq 0$ to the broken symmetry phase. Since $\gamma$ enters the potential (7) only through the $\cos 3 \gamma$ dependence in the cubic term, the minimization in this variable can be performed separately. The global minimum is either at $\gamma_{m}=0(2 \pi / 3$, $4 \pi / 3)$ for $A_{3}<0$ or at $\gamma_{m}=\pi / 3(\pi, 5 \pi / 3)$ for $A_{3}>0$. The second possibility can be expected via changing the sign of the corresponding $\beta_{m}$ and simultaneously setting $\gamma_{m}=0$.

The IBM phase can be described as follows:

1. For $A_{3}^{2}<4 A_{2}\left|A_{4}\right|$, phase with $\beta_{m}=0$ interpreted as spherical $\mathrm{U}(5)$ dynamical symmetry.

2. For $A_{3}^{2}>4 A_{2}\left|A_{4}\right| ; A_{3}<0$, phase with $\beta_{m}>0, \gamma_{m}=0$ interpreted as prolate deformed $\mathrm{SU}(3)$ dynamical symmetry.

3. For $A_{3}^{2}>4 A_{2}\left|A_{4}\right| ; A_{3}>0$, phase with $\beta_{m}>0, \gamma_{m}=$ $\pi / 3$ interpreted as oblate deformed $S U \overline{(3)}$ dynamical symmetry.

4. The dynamical symmetries $\mathrm{O}(6)$ and $\bar{O}(6)$ do not represent separate phases.

For $\beta$ non-zero, the first derivative of Eq. (7) must be zero and the second derivative positive. This gives

$$
\begin{array}{r}
\beta^{3}\left(4 A_{4} \beta^{2}+3 A_{3} \beta \cos 3 \gamma+2 A_{2}\right)=0 \\
12 A_{4} \beta^{2}+6 A_{3} \beta \cos 3 \gamma+2 A_{2}>0
\end{array}
$$

The solution for $\beta \neq 0$ are obtained if we set $A_{3}=0$ in equation (9). Then equation (9) gives

$$
4 A_{4} \beta^{2}+2 A_{2}=0
$$

or

$$
\beta= \pm \sqrt{\frac{-A_{2}}{2 A_{4}}}
$$

This requires $A_{4}$ and $A_{2}$ to have opposite signs. Since $A_{4}$ must be positive for bound solutions, $A_{2}$ must be negative in deformed phase.

The finite $\beta$ solutions corresponds to prolate $(\beta>0)$ and oblate $(\beta<0)$ equilibrium shapes, separated by a curve corresponding to $A_{3}=0$. Thus, there are in total, three phases. The spherical-deformed phase transition is generated by a change in sign of $A_{2}$, while the prolate-oblate correspond to changing the sign of $A_{3}$.

For $\gamma=0$ (to study the $\beta$ dependence) and in the large $N$-limit (i.e., $N-1=N$ ), yield the following coefficients in the PESs

$$
\begin{aligned}
& A_{2}=\left[\epsilon+4 a_{2} N\right] N \\
& A_{3}=-4 \sqrt{\frac{2}{7}} \chi a_{2} N^{2} \\
& A_{4}=\left[\epsilon+\frac{2}{7} \chi^{2} a_{2} N\right] N
\end{aligned}
$$



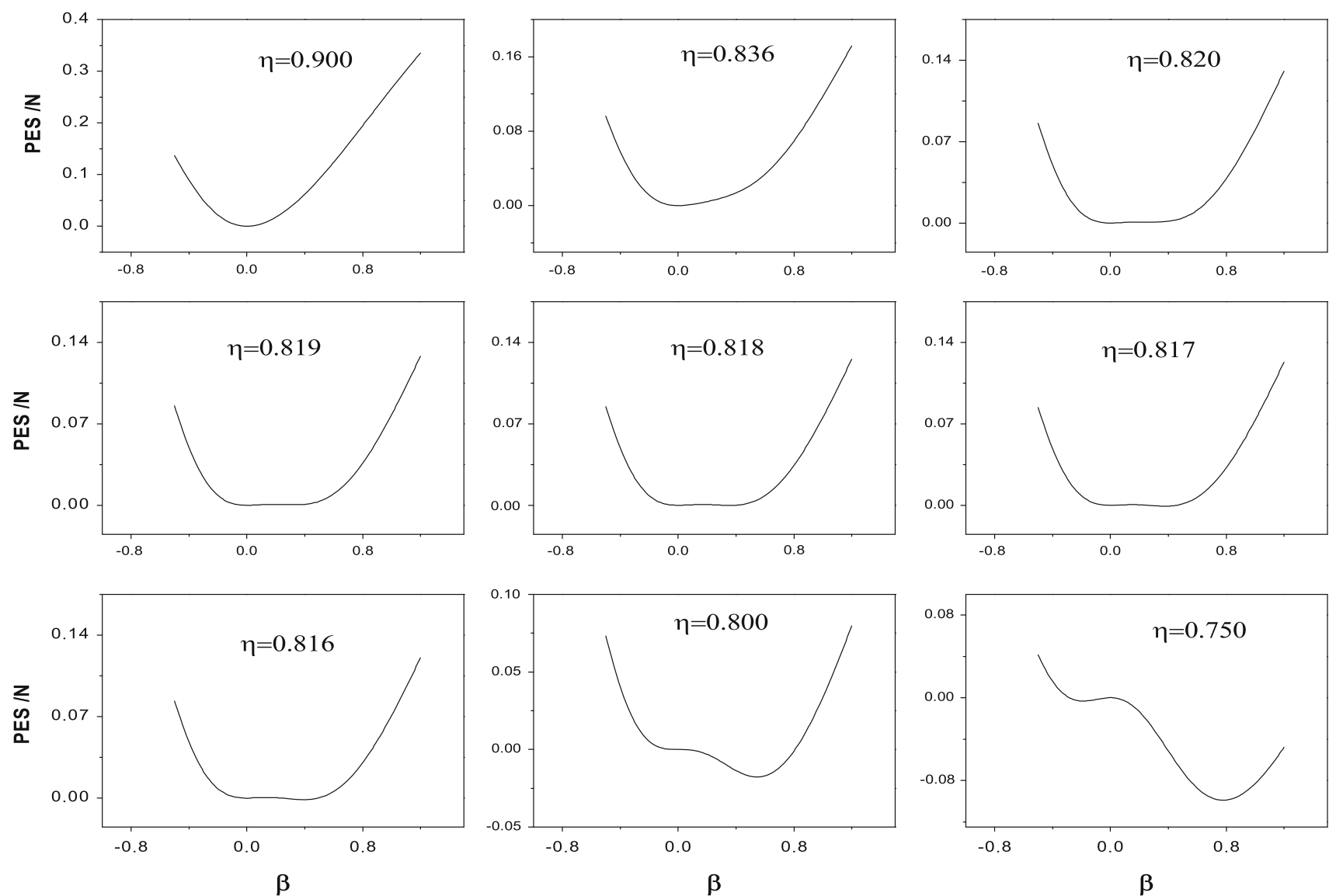

Fig. 1 Potential energy surface (PES) as a function of deformation parameters $\beta$ corresponding to U(5)-SU(3) transition for large $N$ limit for different values of control parameter $\eta$
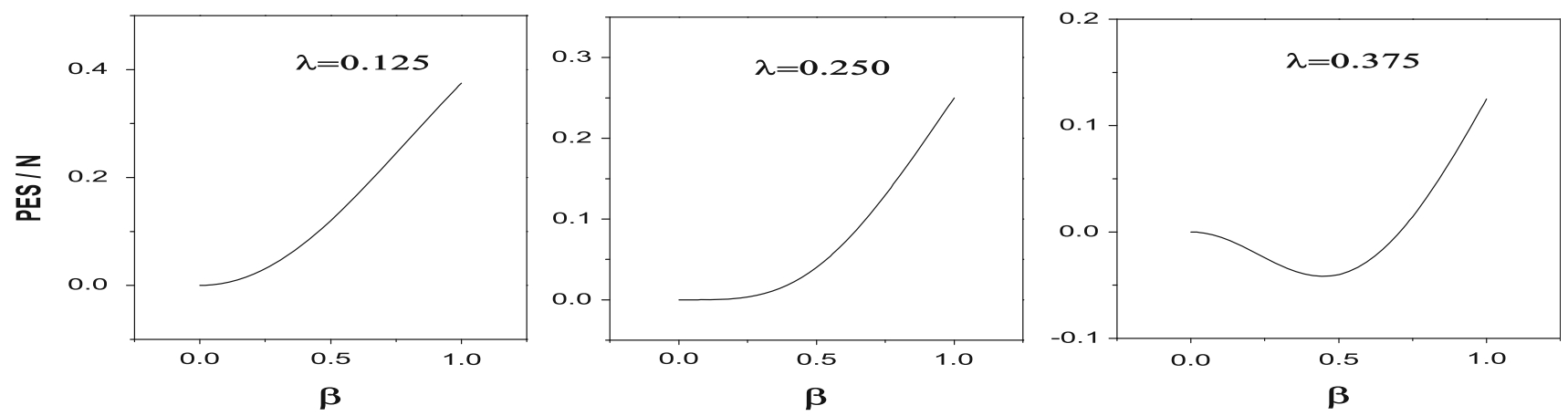

Fig. 2 Potential energy surface (PES) as a function of deformation parameters $\beta$ corresponding to $\mathrm{U}(5)-\mathrm{O}(6)$ transition for large $N$ limit for three values of control parameter $\lambda$

providing that $A_{2}>0$ and $A_{3}>0$, then the critical point is located at $A_{3}^{2}=4 A_{2} A_{4}$, which leads to

$$
1=-\frac{a_{2} N}{\epsilon}\left(4+\frac{2}{7} \chi^{2}\right)
$$

If we introduce the control parameter $\eta$ such that

$\frac{1-\eta}{\eta}=-\frac{a_{2} N}{\epsilon}$
Then the critical point is located at

$\eta_{c}=\frac{4+\frac{2}{7} \chi^{2}}{5+\frac{2}{7} \chi^{2}}$

At $\eta_{c}$ the depth of the $\beta=0$ and $\beta \neq 0$ minima is equal.

The antispinodal point for the transition U(5)-SU(3) appears when $A_{2}=0$, i.e.,

$\frac{-a_{2} N}{\epsilon}=\frac{1}{4}$ 
or

$\eta=4 / 5$

For $\chi=-\sqrt{7} / 2$, the critical point is located at $\eta_{c}=9 / 11$ and the equilibrium value of the deformation parameter at the critical point is $\beta_{0}=1 / 2 \sqrt{2}$. At $\eta=1$ the system is in

Table 1 The optimized fitted parameters for the Gd isotopic chain

\begin{tabular}{lrrll}
\hline Nucleus & \multicolumn{1}{l}{$N_{B}$} & \multicolumn{1}{l}{$A_{2}$} & \multicolumn{1}{l}{$A_{3}$} & $A_{4}$ \\
\hline${ }^{150} \mathrm{Gd}$ & 9 & 4.0693 & -1.6933 & 6.1707 \\
${ }^{152} \mathrm{Gd}$ & 10 & 2.4414 & -2.6035 & 5.6722 \\
${ }^{154} \mathrm{Gd}$ & 11 & -0.9785 & -3.460 & 4.4119 \\
${ }^{156} \mathrm{Gd}$ & 12 & -3.9732 & -3.6407 & 3.5284 \\
${ }^{158} \mathrm{Gd}$ & 13 & -5.1200 & -3.8485 & 3.4606 \\
${ }^{160} \mathrm{Gd}$ & 14 & -7.8990 & -3.0936 & 2.8020 \\
${ }^{162} \mathrm{Gd}$ & 15 & -12.4621 & -2.2000 & 1.1697 \\
\hline
\end{tabular}

the symmetric phase since the PES has a unique minimum at $\beta=0$. When $\eta$ decreases, one reaches the spinodal point at $\eta<\eta_{c}(\eta=0.820)$ where a second local minimum arises.

The two minima have the same depth at the critical point $\eta_{c}=9 / 11$ (0.818). Beyond this value for $\eta>\eta_{c}$ the symmetric minimum at $\beta=0$ becomes a local minimum till $\eta=4 / 5(0.800)$ where it becomes antispinodal point. A sketch of this evolution is illustrated in Fig. 1.

For $\chi=0\left(A_{3}=0\right)$, and for large $N$ limit the PES reduces to

$$
E(N, \beta)=\frac{A_{2} \beta^{2}+A_{4} \beta^{4}}{\left(1+\beta^{2}\right)^{2}}
$$

with

$$
\begin{aligned}
& A_{2}=\left(\epsilon+4 a_{2} N\right) N \\
& A_{4}=\epsilon N
\end{aligned}
$$

At the critical point $A_{2}=0$, which leads to
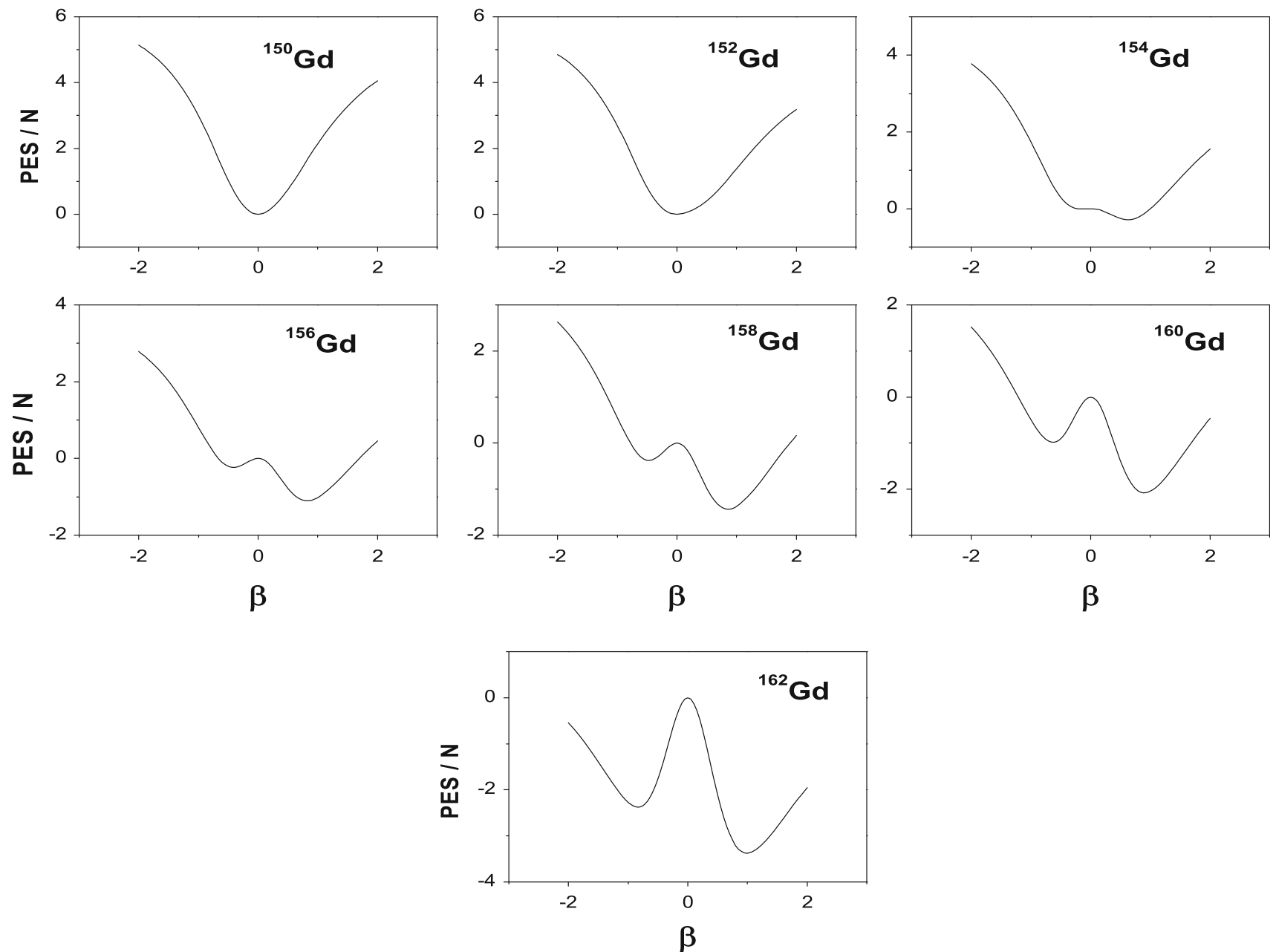

Fig. 3 Potential energy curves as a function of deformation parameter $\beta$ in the U(5)-SU(3) transition for the even-even Gd isotopic chain obtained from IBM with intrinsic state formalism. The total number of bosons $N=9-15$ and $\chi=-\sqrt{7} / 2$ 

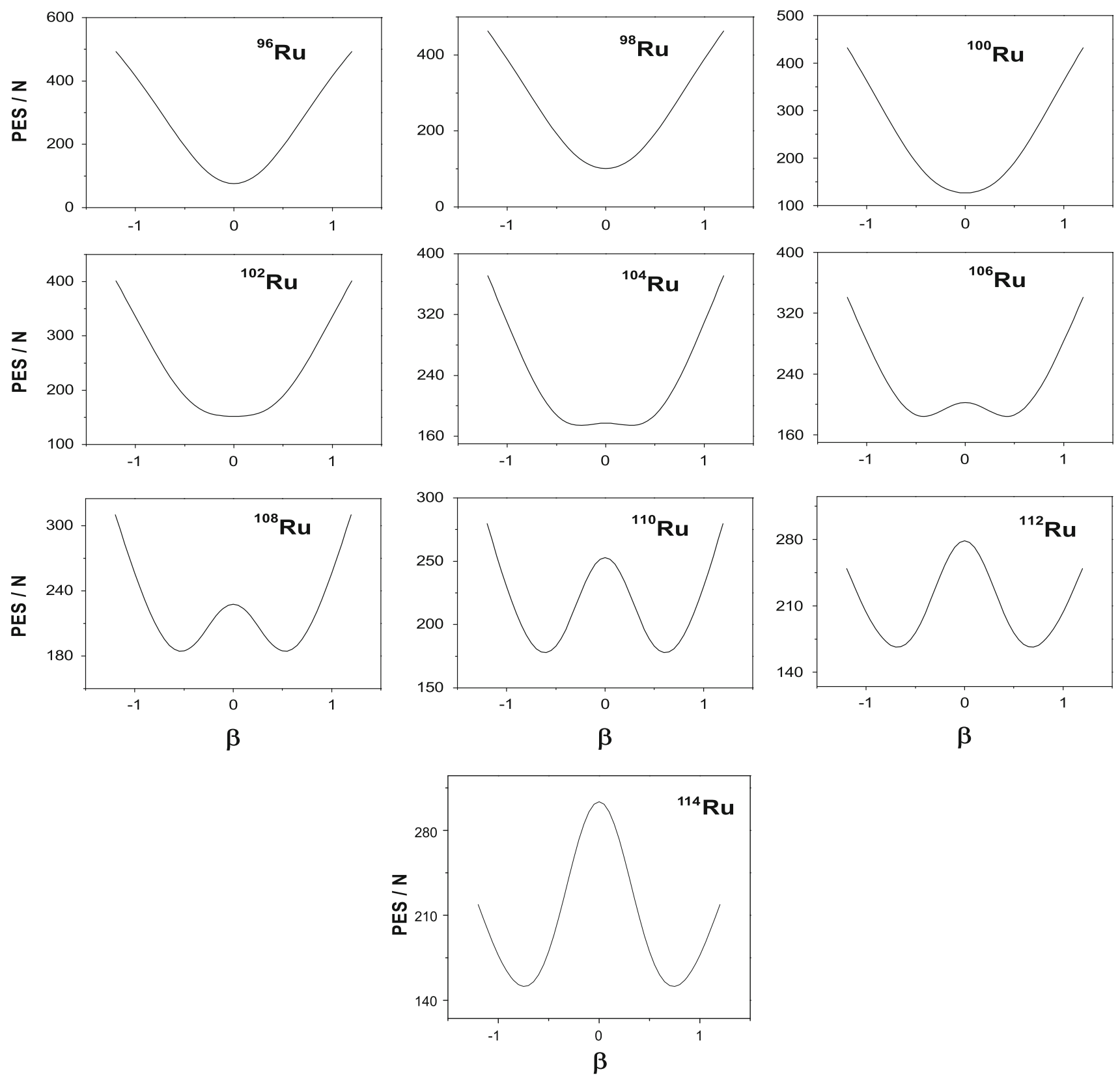

Fig. 4 The same as in Fig. 3 but for $\mathrm{U}(5)-\mathrm{O}(6)$ transition for the even-even Ru isotopics chain. The total number of bosons $N=4-13$ and $\chi=0$

$$
-\frac{a_{2} N}{\epsilon}=\frac{1}{4}
$$

or

$\frac{1-\eta}{\eta}=\frac{1}{4}$

Then the critical point is located at $\eta_{c}=4 / 5$. All the three points, spinodal, critical and antispinodal are located at $\eta=4 / 5$ and the quantum phase transition between spherical and deformed phase is of the second order. A sketch of this case is illustrated in Fig. 2.

\section{Application of the model to the Gd and Ru isotopic chains}

We used the $\chi^{2}$ test to get the coefficients $A_{1}, A_{2}$ and $A_{3}$ of the PESs. The $\chi^{2}$ function is defined in the standard way,

$\chi^{2}=\left[\frac{1}{N_{\text {data }}-N_{\text {par }}} \sum_{i=1}^{N_{\text {data }}}\left(\frac{\chi_{i}^{\exp }(I)-\chi_{i}^{\mathrm{IBM}}}{\sigma_{i}}\right)\right]^{2}$

where $N_{\text {data }}$ is the number of data to be fitted, $N_{\text {par }}$ is the number of parameters used in the IBM fit, $\chi_{i}^{\exp }$ is an experimental energy level [19] or the two-neutron 
Table 2 The optimized fitted parameters for the Ru isotopic chain

\begin{tabular}{lrrrr}
\hline Nucleus & $N_{B}$ & \multicolumn{1}{l}{$A_{2}$} & \multicolumn{1}{l}{$A_{4}$} & $a_{0}$ \\
\hline${ }^{96} \mathrm{Ru}$ & 4 & 527.6 & 831.2 & 75.9 \\
${ }^{98} \mathrm{Ru}$ & 5 & 273.4 & 778.2 & 101.2 \\
${ }^{100} \mathrm{Ru}$ & 6 & 219.2 & 725.2 & 126.5 \\
${ }^{102} \mathrm{Ru}$ & 7 & 65.0 & 672.2 & 151.8 \\
${ }^{104} \mathrm{Ru}$ & 8 & -89.2 & 619.2 & 177.1 \\
${ }^{106} \mathrm{Ru}$ & 9 & -243.4 & 566.2 & 202.4 \\
${ }^{108} \mathrm{Ru}$ & 10 & -397.6 & 513.2 & 227.7 \\
${ }^{110} \mathrm{Ru}$ & 11 & -551.8 & 460.2 & 253.0 \\
${ }^{112} \mathrm{Ru}$ & 12 & -706.0 & 407.2 & 278.3 \\
${ }^{114} \mathrm{Ru}$ & 13 & -860.2 & 354.2 & 303.6 \\
\hline
\end{tabular}

separation energies or the $B(E 2)$ values and $\chi_{i}^{\mathrm{IBM}}$ is the corresponding calculated IBM value and $\sigma_{i}$ is the experimental error assigned to each $\chi_{i}^{e x p}$. To perform the fit, we minimized $\chi^{2}$ function for the selected energy levels $\left(2_{1}^{+}, 4_{1}^{+}, 6_{1}^{+}, 8_{1}^{+}, 0_{2}^{+}, 2_{3}^{+}, 4_{3}^{+}, 2_{2}^{+}, 3_{1}^{+}\right.$and $\left.4_{2}^{+}\right)$using $A_{1}, A_{2}$ and $A_{3}$ as free parameters.

We apply now the formalism outlined in the previous section to $\mathrm{Gd}$ and $\mathrm{Ru}$ isotopic chains. In case of Gadolinium chain ${ }_{64}^{150-162} \mathrm{Gd}$ we assumed the structure parameters $\chi$ of the quadrupole operator to be equal to $-\sqrt{7} / 2$, because some Gd isotopes clearly exhibit the character of the SU(3) dynamical symmetry. Using the optimized fitted parameters shown in Table 1, we calculated the PESs which are illustrated in Fig. 3. The phase diagram exhibits first-order shape phase transition from spherical U(5) to deformed axial symmetric prolate $\mathrm{SU}(3)$ when moving from light isotopes to heavy ones. The ${ }^{150} \mathrm{Gd}$ nucleus still shows a vibrational structure while ${ }^{156-162} \mathrm{Gd}$ are considered as rather good SU(3) examples.

As an example of isotopic chain in which the structure changes from spherical $\mathrm{U}(5)$ to $\gamma$-unstable $\mathrm{O}(6)$ symmetry, we have studied the Ruthenium chain ${ }^{96-114}{ }_{44} \mathrm{Ru}$. The corresponding PESs are plotted in Fig. 4 using the coefficients in Table 2. We considered $\chi=0$ and performed the same process of fitting as applied in Gd chain. The lighter isotopes exhibit a vibrational structure, while the heavier ones present a $\gamma$-unstable behavior with the critical point located of ${ }^{104} \mathrm{Ru}$ isotope.

\section{Conclusion}

In the present work, we have analyzed the shape phase transitions in the framework of a two-parameter simplified truncated sd-IBM1 as a very tractable model for theoretical studies. We used the boson intrinsic coherent state formalism which provides a connection between the IBM and the geometric collective model (GCM) to calculate the PESs. We have analyzed the critical points of the shape phase transitional regions $\mathrm{U}(5)-\mathrm{SU}(3)$ and $\mathrm{U}(5)-\mathrm{O}(6)$ in the space of the model parameters. We have investigated the first-order quantum phase transition from spherical $\mathrm{U}(5)$ to deformed axial symmetric prolate $\mathrm{SU}(3)$ along the chain of ${ }^{150-162} \mathrm{Gd}$ isotopes and the second-order quantum phase transition from spherical $\mathrm{U}(5)$ to $\gamma$-unstable rotor $\mathrm{O}(6)$ along the chain of ${ }^{96-114} \mathrm{Ru}$ isotopes. We find that the critical nuclei characterizing the phase transitions are ${ }^{154} \mathrm{Gd}$ and ${ }^{104} \mathrm{Ru}$. To get the optimized parameter of the PES for each nucleus, a computer-simulated search program is performed to obtain a minimum root mean squared (rms) deviation between calculated and some experimentally selected low-lying energy levels, B(E2) transition rates and two-neutron separation energies.

Open Access This article is distributed under the terms of the Creative Commons Attribution License which permits any use, distribution, and reproduction in any medium, provided the original author(s) and the source are credited.

\section{References}

1. Iachello, F., Arima, A.: The Interacting Boson Model. Cambridge University Press, Cambridge (1987)

2. Khalaf, A.M., Taha, M.M., Kotb, M.: Identical bands and $\Delta \mathrm{I}=2$ staggering in superdeformed nuclei in $\mathrm{A} \sim 150$ mass region using three parameters rotational model. Prog. Phys. 8, 39-44 (2012)

3. Khalaf, A.M., Ismail, A.M.: The nuclear shape phase transitions studied within the geometric collective model. Prog. Phys. 9, 51-55 (2013)

4. Khalaf, A.M., Ismail, A.M.: Structure shape evolution in lanthanide and actinide nuclei. Prog. Phys. 9, 98-104 (2013)

5. Khalaf, A.M., Hamdy, H.S., El Sawy, M.M.: Nuclear shape transition using interacting boson model with the intrinsic coherent state. Prog. Phys. 9, 44-51 (2013)

6. Turner, P.S., Rowe, D.J.: Phase transitions and quasidynamical symmetry in nuclear collective models. II. The spherical vibrator to gamma-soft rotor transition in an $\mathrm{SO}(5)$-invariant Bohr model. Nucl. Phys. A 756, 333-355 (2005)

7. Rowe, D.J., Turner, P.S.: The many relationships between the IBM and the Bohr model. Nucl. Phys. A 760, 59-81 (2005)

8. Cejnar, P., Heinze, S., Dobes, J.: Thermodynamic analogy for quantum phase transitions at zero temperature. Phys. Rev. C 71, 011304(R) (2005)

9. Heinze, S., et al.: Evolution of spectral properties along the $\mathrm{O}(6)-$ $\mathrm{U}(5)$ transition in the interacting boson model. I. Level dynamics. Phys. Rev. C 73, 014306-014316 (2006)

10. Dieperink, A.E.L., Scholten, O., Iachello, F.: Classical limit of the interacting-boson model. Phys. Rev. Lett. 44, 1747-1750 (1980)

11. Ginocchio, J.N.: An exactly solvable anharmonic Bohr hamiltonian and its equivalent boson hamiltonian. Nucl. Phys. A 376, 438-450 (1982)

12. Ginocchio, J.N., Kirson, M.W.: Relationship between the Bohr collective Hamiltonian and the interacting-boson model. Phys. Rev. Lett. 44, 1744 (1980) 
13. Iachello, F.: Dynamic symmetries at the critical point. Phys. Rev. Lett. 85, 3580-3583 (2000)

14. Iachello, F.: Analytic description of critical point nuclei in a spherical-axially deformed shape phase transition. Phys. Rev. Lett. 87, 052502-052506 (2001)

15. Iachello, F.: Phase transitions in angle variables. Phys. Rev. Lett. 91, 132502-132507 (2003)

16. Raduta, A.A., Faessler, A.: A coherent state description of the shape phase transition in even-even Gd isotopes. J. Phys. G Nucl. Part Phys. 31, 873-901 (2005)
17. Garcia-Ramos, J.E., et al.: Phase transitions and critical points in the rare-earth region. Phys. Rev. C 68, 024307 (2003)

18. Frank, A., Alonso, C.E., Aria, J.M.: Search for E(5) symmetry in nuclei: the Ru isotopes. Phys. Rev. C 65, 014301 (2001)

19. Singh, B., Zywina, R., Firestone, R.B.: Table of superdeformed nuclear bands and fission isomers. Nucl. Data Sheets 97, 241-592 (2002) 